\title{
On the micro-macro limit in traffic flow
}

\author{
R.M. Colombo (*) - E. Rossi (**)
}

ABstRACT - We investigate the relations between a macroscopic Lighthill-Whitham and Richards model and a microscopic follow-the-leader model for traffic flow. Solutions to the microscopic model are proved to tend to those to the macroscopic one in a sort of kinetic limit, i.e. as the number of individuals tends to $+\infty$ while their total mass is constant. Based on this convergence result, we approximately compute the solutions to a conservation law by means of the integration of an ordinary differential system.

Mathematics Subject Classification (2010). 35L65, 90B20.

KEYWORDS. Macroscopic traffic models; microscopic traffic models; conservation laws.

\section{Introduction}

Aim of this paper is to investigate the relations between a microscopic and a macroscopic model for traffic flow. More precisely, we consider the Lighthill-Whitham [7] and Richards [10] continuum model

$$
\left\{\begin{array}{l}
\partial_{t} \rho+\partial_{x}(\rho v(\rho))=0 \\
\rho(0, x)=\tilde{\rho}(x)
\end{array}\right.
$$

where $t \in \mathbb{R}^{+}$is time, $x \in \mathbb{R}$ is the space coordinate, $\rho=\rho(t, x)$ is the (average) vehicular density, with $\rho \in[0,1]$, and $v \in \mathbf{C}^{0,1}([0,1] ;[0, V])$ is the macroscopic speed law. At the discrete level, we choose the first-order

(*) Indirizzo dell'A.: INdAM Unit, University of Brescia, via Branze 38, 25123 Brescia, Italy.

E-mail: Rinaldo.Colombo@UniBs.it

(**) Indirizzo dell'A.: Department of Mathematics and Applications, University of Milano-Bicocca, via Cozzi 55, 20125 Milano, Italy.

E-mail: e.rossi50@campus.unimib.it 
Follow the Leader [1] model

$$
\begin{cases}\dot{p}_{i}=w\left(p_{i+1}-p_{i}\right) & i=1, \ldots, n \\ \dot{p}_{n+1}=V & \\ p_{i}(0)=\tilde{p}_{i} & i=1, \ldots, n+1\end{cases}
$$

where $p_{1}, \ldots, p_{n+1}$ are the positions of the $n+1$ drivers, with $p_{i+1}-p_{i} \geq \ell, \ell$ being the average vehicle's length, and $w \in \mathbf{C}^{0,1}([\ell,+\infty[;[0, V])$ is the microscopic speed law. Remark that it is required to assign the speed of the foremost vehicle, but it is not necessary that this speed be the maximal one.

The macroscopic description (1.1) and the microscopic one (1.2) are related through particle paths, which are the trajectories of single individuals according to (1.1), namely the solutions to the ordinary differential equation

$$
\left\{\begin{array}{l}
\dot{p}=v(\rho(t, p(t))) \\
p(0)=\tilde{p},
\end{array}\right.
$$

as $\tilde{p}$ varies in $\mathbb{R}$, see [4] for the well posedness of (1.3). The connection between the macroscopic model (1.2) and the microscopic one (1.1) consists in imposing that each equation in (1.2) is the particle path (1.3) for (1.1). The only exception is the foremost vehicle, whose speed is assigned.

Below, through ad hoc operators, we establish a relation between the macroscopic variable $\rho$ and the microscopic one $\left(p_{1}, \ldots, p_{n+1}\right)$, showing that the two descriptions are to some extent specular. Then, we show that solutions to (1.1) tend to solutions to (1.2) in a sort of kinetic limit, i.e. as the number of individuals tends to $+\infty$ while $n \ell$ remains constant.

From the modelling point of view, this result justifies the LighthillWhitham and Richards model (1.1) as the limit of a first order follow the leader model, as the number of individuals tends to $+\infty$. For a related approach with emphasis on the Hamilton-Jacobi equation we refer to [5].

Besides, the limiting procedure outlined above suggests the use of the ordinary differential equations (1.2) also as a tool for the numerical integration of the partial differential equation (1.1), see Section 3. This possibility is thoroughly investigated below. Several integrations illustrate the rigorous results. However, a numerical algorithm to compute the solutions to (1.1) based on the numerical solution to the ordinary differential system (1.2) hardly competes with an ad hoc method, such as the classical Lax-Friedrichs method.

The next section presents the main analytical results. Section 3 is devoted to several numerical integrations of both (1.1) and (1.2). All technical details are deferred to the final Section 4 . 


\section{Analytical Result}

We denote below $\mathbb{R}^{+}=[0,+\infty[$.

In the sequel, we consider $n+1$ vehicles of total mass $m$, and let $\ell_{n}=m / n$. To formalize the connection between the macro- and microscopic descriptions, we introduce the natural spaces for the macroscopic density $\rho$ and for the individual drivers' positions $\boldsymbol{p}=\left(p_{1}, \ldots, p_{n+1}\right)$

$$
\begin{aligned}
& R_{m}=\left\{\rho \in \mathbf{L}^{1}(\mathbb{R} ;[0,1]): \int_{\mathbb{R}} \rho(x) \mathrm{d} x=m \text { and spt } \rho \text { is compact }\right\} \\
& P_{n}=\left\{\boldsymbol{p} \in \mathbb{R}^{n+1}: p_{i+1}-p_{i} \geq \ell_{n}, \quad \forall i=1, \ldots, n\right\}
\end{aligned}
$$

and the operators that implement it, namely

$$
\begin{aligned}
& E_{n}: \quad R_{m} \rightarrow P_{n} \\
& \rho \rightarrow\left\{\begin{aligned}
p_{n+1} & =\max (\operatorname{spt} \rho) \\
p_{i} & =\max \left\{p \in \mathbb{R}: \int_{p}^{p_{i+1}} \rho(x) \mathrm{d} x=\ell_{n}\right\} \quad i=1, \ldots, n .
\end{aligned}\right. \\
& C_{n}: P_{n} \rightarrow R_{m} \\
& \boldsymbol{p} \rightarrow \sum_{i=1}^{n} \frac{\ell_{n}}{p_{i+1}-p_{i}} \chi_{\left[p_{i}, p_{i+1}[\right.}
\end{aligned}
$$

Given a macroscopic density $\rho$, the operator $E_{n}$ provides a corresponding microscopic description through the vector $\boldsymbol{p}=E_{n} \rho$ of the vehicles' positions. On the contrary, given the discrete positions $\boldsymbol{p}$, the operator $C_{n}$ provides a corresponding piecewise constant density $\rho=C_{n} \boldsymbol{p}$.

The next Proposition formalizes the strict correlation between these two maps.

Proposition 2.1. For every $n \in \mathbb{N}$ with $n \geq 2$ and for every $m>0$, $E_{n} \circ C_{n}=\operatorname{Id}_{P_{n}}$. Viceversa, $C_{n} \circ E_{n} \rightarrow \operatorname{Id}_{R_{m}}$ pointwise in $R_{m} \cap \mathbf{B V}(\mathbb{R} ;[0,1])$ with respect to the $\mathbf{L}^{1}$-norm, so that

$\lim _{n \rightarrow+\infty}\left\|\left(C_{n} \circ E_{n}\right)(\rho)-\rho\right\|_{\mathbf{L}^{1}}=0$ for all $\rho \in R_{m} \cap \mathbf{B V}(\mathbb{R} ;[0,1])$ and $\lim _{n \rightarrow+\infty}\left(\left(C_{n} \circ E_{n}\right)(\rho)\right)(x)=\rho(x) \quad$ for all $\rho \in R_{m} \cap \mathbf{B V}(\mathbb{R} ;[0,1])$, for a.e. $x \in \mathbb{R}$. 
The proof is deferred to Section 4. Note that $C_{n} \circ E_{n} \neq \operatorname{Id}_{R_{m}}$. In fact, the image of $C_{n}$ is a subset of $R_{m}$ that contains only piecewise constant functions. Hence, for any $\rho \in R_{m}$ which is not piecewise constant, obviously we have $\rho \neq\left(C_{n} \circ E_{n}\right)(\rho)$. Moreover, $C_{n}$ is Lipschitz continuous with respect to the $\mathbf{L}^{1}$ norm while $E_{n}$ is not continuous. Below, these two properties are not necessary and for their proof we refer to [11, Chapter 1].

While $E_{n}$ and $C_{n}$ provide the link between the macro- and microscopic descriptions, the link between the two models (1.1) and (1.2) is ensured setting

$$
w(\delta)=v\left(\ell_{n} / \delta\right) \quad \text { or, equivalently, } \quad v(r)=w\left(\ell_{n} / r\right) .
$$

We assume throughout the following condition on $v$ :

(V) $v \in \mathbf{C}^{0,1}([0,1] ;[0, V])$ is such that $v^{\prime}(\rho) \leq 0$ for a.e. $\rho \in[0,1], v(0)=V$ for a suitable positive $V$ and $v(1)=0$.

The following propositions show that both (1.1) and (1.2) are well posed, in the sense that these Cauchy problems have solutions for all $t \in \mathbb{R}^{+}$in the due set $R_{m}$ or $P_{n}$.

Proposition 2.2. Let (V) hold. For any $m>0$, for any initial datum $\tilde{\rho} \in R_{m} \cap \mathbf{B V}(\mathbb{R} ;[0,1])$, the Cauchy problem (1.1) admits a unique solution $\rho \in \mathbf{C}^{0,1}\left(\mathbb{R}^{+} ; R_{m}\right)$.

Proposition 2.3. Let (V) and (2.4) hold. For any $n \in \mathbb{N}$, with $n \geq 2$, for any initial datum $\tilde{\boldsymbol{p}} \in P_{n}$, the Cauchy problem (1.2) admits a unique solution $\boldsymbol{p} \in \mathbf{C}^{0,1}\left(\mathbb{R}^{+} ; P_{n}\right)$.

The proof of Proposition 2.2 follows from [2, Theorem 6.3], for the Lipschitz dependence in time we refer to [2, Formula (6.14)]. The proof of Proposition 2.3 is deferred to Section 4.

Now we are able to show that, as the number of vehicles increases to infinity, the solutions to the microscopic model (1.2)-(2.4) converge to those of the macroscopic one (1.1).

THEOREM 2.4. Let (V) hold, fix $T>0$ and choose $\tilde{\rho} \in R_{m} \cap$ $\mathbf{B V}(\mathbb{R} ;[0,1])$. Then, the diagram in Figure 1 commutes.

In other words, define the initial data for the microscopic model setting $\tilde{\boldsymbol{p}}=E_{n} \tilde{\rho}$, as defined in (2.2). Let $\boldsymbol{p}(t)=\left(p_{1}(t), \ldots, p_{n+1}(t)\right)$ be the corre- 


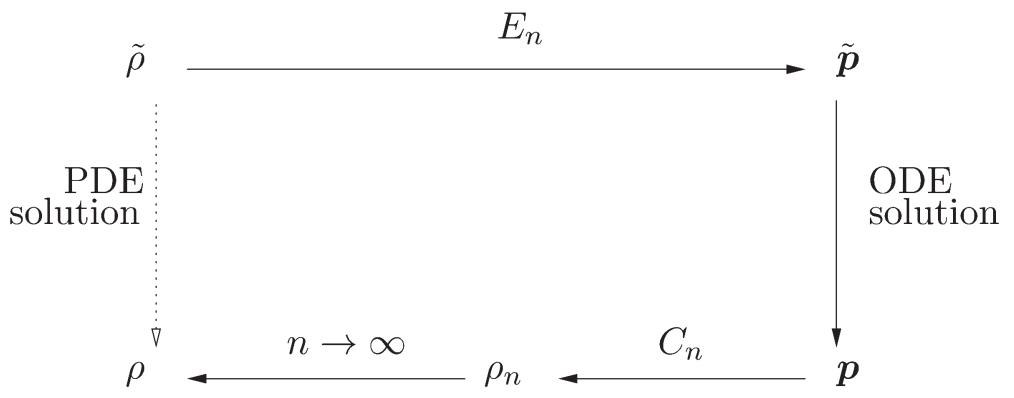

Fig. 1. - Theorem 2.4 proves that the diagram above commutes.

sponding solution to (1.2). Define $\rho_{n}(t)=C_{n} \boldsymbol{p}(t)$, as in (2.3). If there exists $\rho \in \mathbf{L}^{\infty}\left([0, T], R_{m}\right)$ such that

$$
\lim _{n \rightarrow+\infty} \rho_{n}(t, x)=\rho(t, x) \quad \text { a.e. },
$$

then $\rho$ is a weak solution to (1.1) with initial datum $\tilde{\rho}$.

\section{Numerical Integrations}

In the numerical integrations below we use the Greenshield speed law $[12$, p. 6]

$$
v(\rho)=V(1-\rho) \quad \text { with } V>0
$$

and $w$ as in (2.4). The system of ordinary differential equations (1.2) is integrated by means of the Livermore solver [8].

Throughout, to compare the conservation law (1.1) with the microscopic model (1.2)-(2.4) we closely follow the diagram in Figure 1. Given the number $n$ of individuals and an initial datum $\tilde{\rho}$ for the partial differential equation (1.1), we select the corresponding initial datum $\tilde{\boldsymbol{p}}=E_{n}(\tilde{\rho})$ for (1.2). In all the figures, to compare the solutions $\boldsymbol{p}(t)$ to (1.2) and $\rho(t)$ to (1.1), we display $C_{n}(\boldsymbol{p}(t))$ and $\rho(t)$ on the same axis.

Times of integrations and computational domains are selected so that for the chosen initial data and speed laws no wave in the solution to (1.1) hits any numerical boundary.

\section{1 - The Kinetic Limit}

Following the analytical study above, we use initial data for (1.1) supported in a compact interval $[-K, K]$, for a suitable large $K$, as re- 
quired in (2.1). To focus on a shock and a rarefaction wave, we choose the initial data and a visualization domain such that spurious waves are not seen all during the integration time. Below, the analytical expression of the exact solution $\rho=\rho(t)$ is easily available and is used to evaluate the $\mathbf{L}^{1}$-distance

$$
\left\|\rho(T)-C_{n}(\boldsymbol{p}(T))\right\|_{\mathbf{L}^{1}}
$$

Consider first the shock wave originated by the initial datum below:

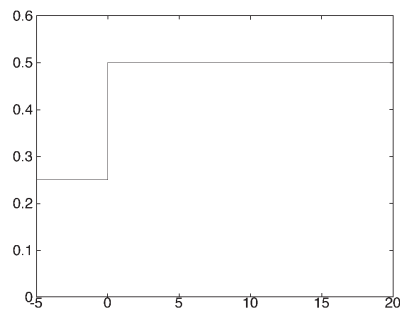

$$
\tilde{\rho}_{S}(x)= \begin{cases}1 / 4 & \text { if } x \in[-K, 0[ \\ 1 / 2 & \text { if } x \in[0, K] .\end{cases}
$$

The numerical integration displayed in Figure 2 qualitatively illustrates the convergence proved in Theorem 2.4: as $n \rightarrow+\infty$, the distance in (3.2) vanishes.

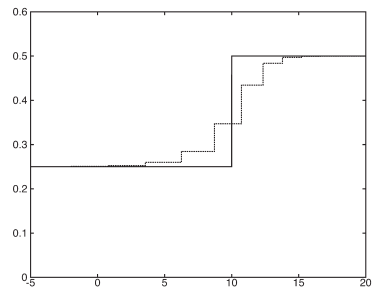

(a) $n=50$

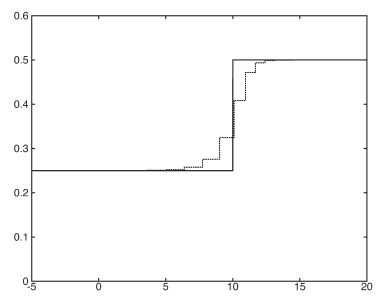

(b) $n=100$

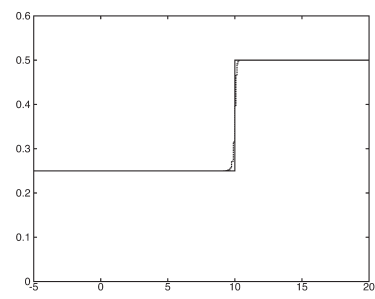

(c) $n=1000$

Fig. 2. - The solid line is the exact solution to the partial differential equation (1.1)-(3.1)-(3.3) at time $T$. The dashed line is $C_{n}(\boldsymbol{p}(T))$, for different values of the vehicles' number $n$. Here, $T=2$ and $V=20$.

A quantitative information is provided in Figure 3, left, where the quantity (3.2) is plotted as a function of the number $n$ of individuals in a logarithmic scale. Clearly, the convergence rate is exponential with the number of vehicles. We observe that the integration time of the microscopic model (1.2)-(2.4)-(3.1) also grows exponentially with the number of individuals, see Figure 3, right. 

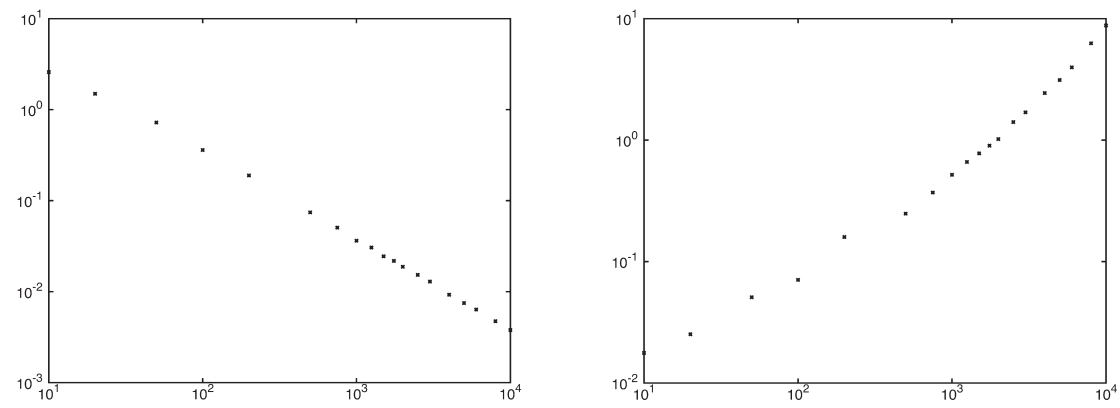

Fig. 3. - Left, the $\mathbf{L}^{1}$-distance (3.2) as a function of $n$ and, right, the integration time as a function of $n$. Both diagrams are in logarithmic scale.

Now, consider the rarefaction wave originated by the initial datum:

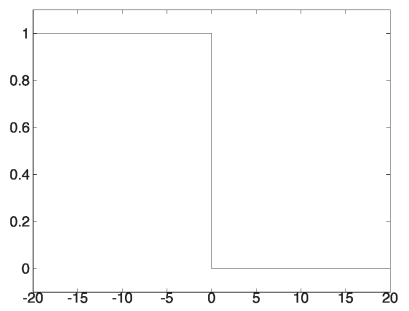

$$
\tilde{\rho}_{R}(x)= \begin{cases}1 & \text { if } x \in[-K, 0[ \\ 0 & \text { if } x \in[0, K],\end{cases}
$$

which corresponds to the standard situation of a traffic light turning green at time $t=0$. A qualitative illustration of the limit in Theorem 2.4 is in Figure 4 , for $n=50,100,1000$. The quantitative evaluation of the distance (3.2) in the case of the rarefaction (3.4) is in Figure 5. Again, the convergence is clearly exponential.

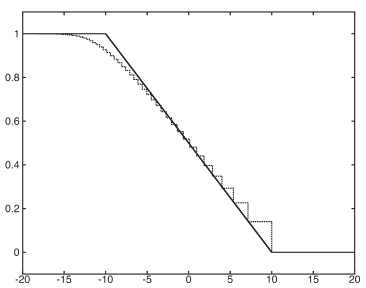

(a) $n=50$

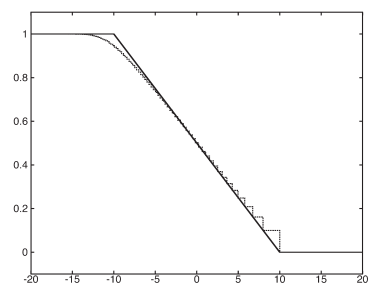

(b) $n=100$

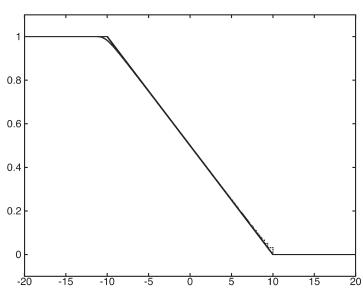

(c) $n=1000$

Fig. 4. - The solid line is the exact solution to the partial differential equation (1.1)-(3.1)-(3.4) at time $T$. The dashed line is $C_{n}(\boldsymbol{p}(T))$, for different values of the vehicles' number $n$. Here, $T=1$ and $V=10$. 

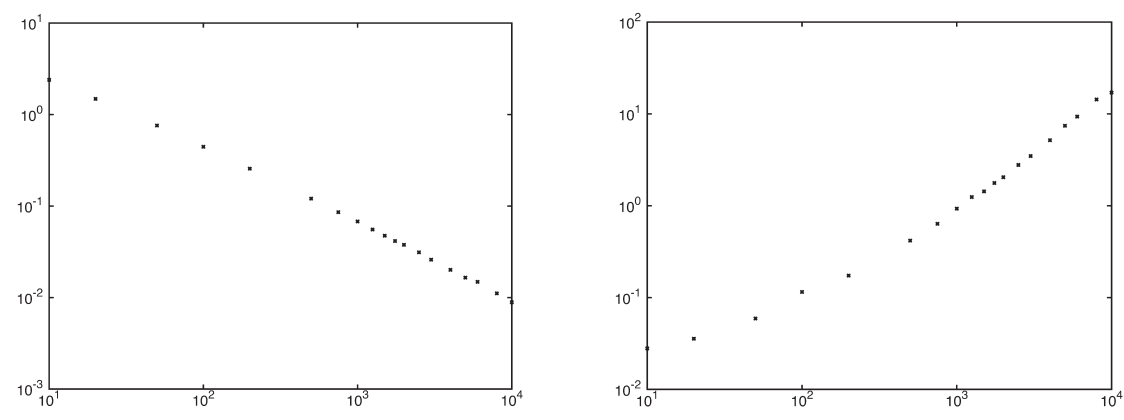

Fig. 5. - Left, the $\mathbf{L}^{1}$-distance (3.2) as a function of $n$ and, right, the integration time as a function of $n$. Both diagrams are in logarithmic scale.

\section{2 - ODEs as an Algorithm to Integrate PDEs}

This paragraph is devoted to evaluate the use of the ordinary differential system (1.2) as an algorithm to obtain approximate solutions to the partial differential equation (1.1), relying on Theorem 2.4. To this aim, we consider the following initial datum with compact support, for which the analytical solution is not easily available:

$$
\tilde{\rho}(x)=\left\{\begin{array}{cl}
\frac{x^{2}}{4} & \text { if } 0 \leq x<2 \\
1 & \text { if } 2 \leq x<3 \\
\frac{-x^{2}+6 x-5}{4} & \text { if } 3 \leq x<5 \\
0 & \text { if } 5 \leq x \leq 20 .
\end{array}\right.
$$

Then, we compute the approximate solution $\rho_{\Delta x}=\rho_{\Delta x}(t)$ to (1.1)-(3.5) by means of the standard Lax-Friedrichs algorithm [6, Chapter 4], for different choices of the space mesh size $\Delta x$. Similarly, the initial datum (3.5) is transformed into an initial datum $E_{n} \tilde{\rho}$ for (1.2), for different numbers $n$ of individuals. This latter equation is numerically integrated and its solution $\boldsymbol{p}_{n}=\boldsymbol{p}_{n}(t)$, following the scheme in Figure 1, is transformed back into the density $C_{n}\left(\boldsymbol{p}_{n}(t)\right)$.

To evaluate the errors in the two approximate solutions, we take as a reference "exact" solution to (1.1)-(3.5) an approximate solution $\rho=\rho(t)$ computed by means of the Lax-Friedrichs scheme with a very fine mesh, namely $\Delta x=1 E-4$. More precisely, the errors considered below are the 
supremum on $[0, T]$ of the relative error in the $\mathbf{L}^{1}$-norm, namely

$$
\max _{t \in[0, T]} \frac{\left\|\rho_{\Delta x}(t)-\rho(t)\right\|_{\mathbf{L}^{1}}}{\|\rho(t)\|_{\mathbf{L}^{1}}} \quad \text { and } \max _{t \in[0, T]} \frac{\left\|C_{n}\left(\boldsymbol{p}_{n}(t)\right)-\rho(t)\right\|_{\mathbf{L}^{1}}}{\|\rho(t)\|_{\mathbf{L}^{1}}}
$$

Since $\rho$ is the solution to a conservation law, remark that the quantity $\|\rho(t)\|_{\mathbf{L}^{1}}$ in the denominator above is constant in time and only acts as a scale factor.

Table 1 takes solutions $\rho_{\Delta x}$ and $C_{n} \boldsymbol{p}_{n}$ which have similar times of execution and compares the corresponding errors, measured as in (3.6), for different values of the space mesh size $\Delta x$ and of the vehicles' number $n$.

Solutions obtained with similar execution times have an error, measured as in (3.6), that differs up to about one order of magnitude, the solution $\rho_{\Delta x}$ being more precise. However, Figure 6 shows that the qualitative properties of the solutions are well captured by both algorithms.

Moreover, in Figure 7, we display the absolute error as a function of $x$, estimated as the absolute value of the distance between the solution to the ordinary differential equation (1.2) and the "exact" solution. The different

TABLE 1. - Comparison between the Lax-Friedrichs solution $\rho_{\triangle x}$ to (1.1)-(3.5) and the solution $C_{n} \boldsymbol{p}_{n}$ coming from the system (1.2), obtained with comparable computing times. The corresponding key parameters $\Delta x$ and $n$ and the errors, measured as in (3.6), are also displayed.

\begin{tabular}{|l|c|c||c|c|c|}
\hline \multirow{2}{*}{$\begin{array}{c}\text { Space mesh } \\
\text { size } \Delta x\end{array}$} & Error & \multicolumn{2}{|c|}{ Execution Time } & Error & \multirow{2}{*}{$\begin{array}{c}\text { Number of } \\
\text { vehicles } n\end{array}$} \\
\cline { 2 - 5 } & \multicolumn{2}{|c|}{ Lax-Friedrichs } & \multicolumn{2}{c|}{ ODE system } & 20 \\
\hline 0,02 & $1,32 \mathrm{E}-2$ & 0,06864 & 0,06262 & $1,51 \mathrm{E}-1$ & 100 \\
\hline 0,01 & $6,73 \mathrm{E}-3$ & 0,1585 & 0,1910 & $4,23 \mathrm{E}-2$ & 150 \\
\hline 0,0064 & $4,22 \mathrm{E}-3$ & 0,2737 & 0,2930 & $2,87 \mathrm{E}-2$ & 200 \\
\hline 0,005 & $3,23 \mathrm{E}-3$ & 0,3963 & 0,4242 & $2,17 \mathrm{E}-2$ & 225 \\
\hline 0,0045 & $2,76 \mathrm{E}-3$ & 0,4558 & 0,4877 & $1,66 \mathrm{E}-2$ & 250 \\
\hline 0,004 & $2,51 \mathrm{E}-3$ & 0,5365 & 0,5423 & $1,61 \mathrm{E}-2$ & 500 \\
\hline 0,0025 & $1,57 \mathrm{E}-3$ & 1,080 & 1,216 & $8,95 \mathrm{E}-3$ & 600 \\
\hline 0,002 & $1,20 \mathrm{E}-3$ & 1,527 & 1,545 & $7,30 \mathrm{E}-3$ & 600 \\
\hline 0,0016 & $9,93 \mathrm{E}-4$ & 2,190 & 2,085 & $5,76 \mathrm{E}-3$ & 8,3 \\
\hline 0,001 & $5,56 \mathrm{E}-4$ & 4,867 & 4,551 & $3,41 \mathrm{E}-3$ & 1500 \\
\hline
\end{tabular}



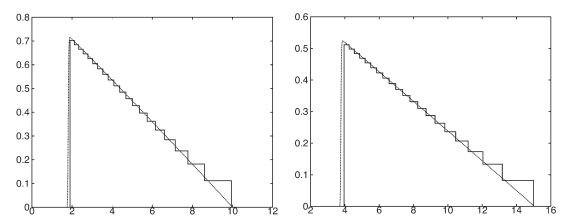

(a) $n=20$ and $\Delta x=0.02$.
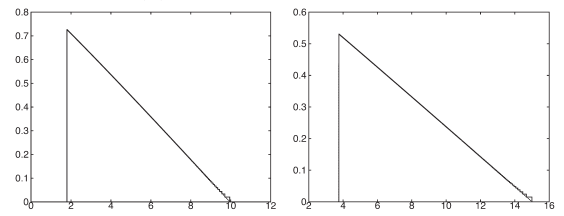

(c) $n=600$ and $\Delta x=0.002$.
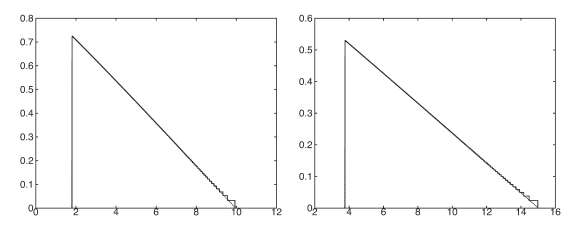

(b) $n=250$ and $\Delta x=0.004$.
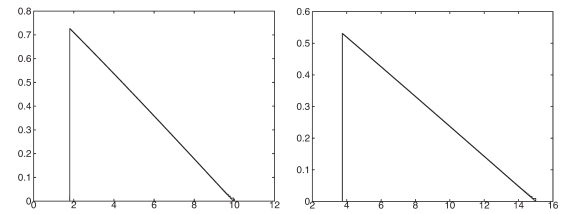

(d) $n=1500$ and $\Delta x=0.001$.

Fig. 6. - Approximate solutions to the Cauchy Problem (1.1)-(3.5) and to the corresponding ordinary differential system (1.2) with datum $E_{n} \tilde{\rho}$, compared for different values of the space mesh $\Delta x$ and of the number of individuals $n$. Each graph displays solutions obtained with the two algorithms in comparable computing times, at $t=0.5$ and $t=1$.
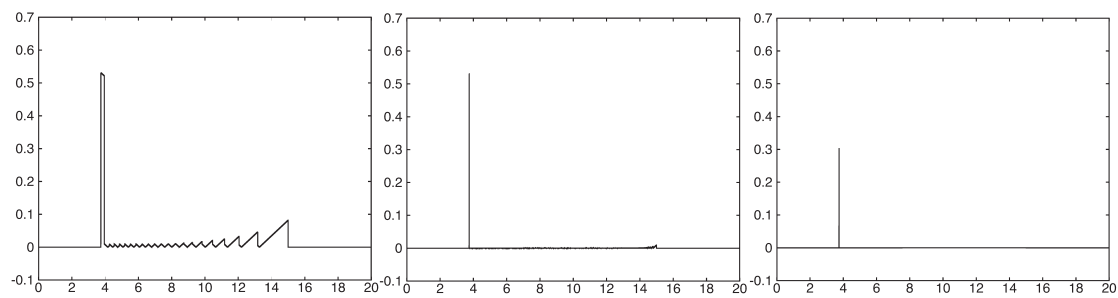

Fig. 7. - The first two diagrams from the left show the absolute error between the solution to (1.2) and the "exact" solution to (1.1)-(3.5), for $n=20$ and $n=1500$. For a comparison, the rightmost diagram is the usual one of the absolute error between the approximate solution to (1.1)-(3.5) and the "exact" solution, with mesh size $\Delta x=0.001$. All graphs refer to time $t=1$.

behaviour of this algorithm from the usual Lax-Friedrichs one is understood comparing the two diagrams on the left of Figure 7 with the rightmost one. The latter graph displays the usual absolute error caused by the Lax-Friedrichs scheme.

Below, for these two algorithms, we also compare the execution times necessary to obtain comparable errors, computed as in (3.6), for different values of the mesh size $\Delta x$ and of the vehicles' number $n$, see Table 2 . In order to obtain comparable errors between the numerical solution to 
TABLE 2. - Comparison between the execution times in solutions having comparable errors, through the Lax-Friedrichs method applied to (1.1) and through the integration of (1.2). The errors are computed as in (3.6).

\begin{tabular}{|c|c|c|c|c|c|}
\hline \multirow{2}{*}{$\begin{array}{l}\text { Space mesh } \\
\text { size } \Delta x\end{array}$} & Time & \multicolumn{2}{|c|}{ Error } & Time & \multirow{3}{*}{$\begin{array}{c}\text { Number of } \\
\text { vehicles } n\end{array}$} \\
\hline & \multicolumn{2}{|c|}{ Lax-Friedrichs } & \multicolumn{2}{|c|}{ ODE system } & \\
\hline 0,05 & 0,02611 & $3,08 \mathrm{E}-2$ & $2,87 \mathrm{E}-2$ & 0,2930 & \\
\hline 0,032 & 0,04097 & $2,04 \mathrm{E}-2$ & $2,17 \mathrm{E}-2$ & 0,4242 & 200 \\
\hline 0,025 & 0,05409 & $1,60 \mathrm{E}-2$ & $1,61 \mathrm{E}-2$ & 0,5423 & 250 \\
\hline 0,002 & 0,06864 & $1,32 \mathrm{E}-2$ & $1,27 \mathrm{E}-2$ & 0,9298 & 400 \\
\hline 0,016 & 0,08786 & $1,07 \mathrm{E}-2$ & $1,06 \mathrm{E}-2$ & 0,8723 & 375 \\
\hline 0,001 & 0,1585 & $6,73 \mathrm{E}-3$ & $6,23 \mathrm{E}-3$ & 1,919 & 750 \\
\hline 0,008 & 0,2097 & $5,25 \mathrm{E}-3$ & $4,99 \mathrm{E}-3$ & 2,818 & 1000 \\
\hline 0,005 & 0,3963 & $3,23 \mathrm{E}-3$ & $3,41 \mathrm{E}-3$ & 4,551 & 1500 \\
\hline 0,0045 & 0,4558 & $2,76 \mathrm{E}-3$ & $2,77 \mathrm{E}-3$ & 6,5346 & 2000 \\
\hline 0,0025 & 1,080 & $1,57 \mathrm{E}-3$ & $1,39 \mathrm{E}-3$ & 21,80 & 5000 \\
\hline 0,00125 & 3,303 & $7,56 \mathrm{E}-4$ & $6,94 \mathrm{E}-4$ & 63,94 & 10000 \\
\hline
\end{tabular}

the ODE system and the numerical solution to the PDE system, the computing time needed by the algorithm that exploits (1.2) to solve (1.1) can be also about ten times longer than that necessary for usual LaxFriedrichs solutions.

\section{Technical Details}

Throughout this section, $n \in \mathbb{N}$ and $n \geq 2$. We refer to [11] for fully detailed proofs.

LemMa 4.1. For every $m>0$, if $\rho \in R_{m}$, then $\operatorname{TV}\left(\left(C_{n} \circ E_{n}\right)(\rho)\right) \leq \operatorname{TV}(\rho)$.

Proof. Setting $\boldsymbol{p}=E_{n}(\rho)$, for every $i \in\{1, \ldots, n\},\left(C_{n} \circ E_{n}\right)(\rho)$ is constant in $\left[p_{i}, p_{i+1}[\right.$ and, by (2.2) and (2.3),

$$
\int_{p_{i}}^{p_{i+1}} \rho(x) \mathrm{d} x=\int_{p_{i}}^{p_{i+1}}\left[\left(C_{n} \circ E_{n}\right)(\rho)\right](x) \mathrm{d} x,
$$


hence

$$
\begin{aligned}
& x \in\left[p_{i}, p_{i+1}\left[\Rightarrow \operatorname{essinf}_{\left[p_{i}, p_{i+1}[\right.} \rho \leq\left[\left(C_{n} \circ E_{n}\right)(\rho)\right](x) \leq \underset{\left[p_{i}, p_{i+1}[\right.}{\operatorname{ess} \sup \rho},\right.\right. \\
& x \in\left[p_{i-1}, p_{i+1}\left[\Rightarrow \operatorname{essinf}_{\left[p_{i-1}, p_{i+1}[\right.}^{\operatorname{ess}} \rho \leq\left[\left(C_{n} \circ E_{n}\right)(\rho)\right](x) \leq \operatorname{ess~sup}_{\left[p_{i-1}, p_{i+1}[\right.} \rho .\right.\right.
\end{aligned}
$$

Therefore,

$$
\begin{aligned}
& \operatorname{TV}\left(\left(C_{n} \circ E_{n}\right)(\rho)\right)=\sum_{i=1}^{n+1}\left|\left[\left(C_{n} \circ E_{n}\right)(\rho)\right]\left(p_{i}+\right)-\left[\left(C_{n} \circ E_{n}\right)(\rho)\right]\left(p_{i}-\right)\right| \\
& \leq \underset{\left[p_{1}, p_{2}[\right.}{\operatorname{ess} \sup _{1}} \rho+\sum_{i=2}^{n}\left(\operatorname{ess~sup}_{\left[p_{i-1}, p_{i+1}[\right.} \rho-\operatorname{essinf}_{\left[p_{i-1}, p_{i+1}[\right.} \rho\right)+\operatorname{ess~sup~}_{\left[p_{n}, p_{n+1}[\right.} \rho \\
& \leq \operatorname{TV}(\rho)
\end{aligned}
$$

completing the proof.

Lemma 4.2. For every $m>0$, fix $\rho \in R_{m}$. For every measurable set $E \subseteq \mathbb{R}$ of finite measure

$$
\lim _{n \rightarrow+\infty} \int_{E}\left[\left(C_{n} \circ E_{n}\right)(\rho)\right](x) \mathrm{d} x=\int_{E} \rho(x) \mathrm{d} x .
$$

Proof. Let $\boldsymbol{p}=E_{n}(\rho)$ and $\rho_{n}=\left(C_{n} \circ E_{n}\right)(\rho)$. The proof consists of several steps.

(I) $E$ is an interval, with extreme points $a$ and $b$.

Remark that $\operatorname{spt} \rho=\left[p_{1}, p_{n+1}\right]$. The continuous and non-decreasing functions

$$
\mathcal{R}(x)=\int_{-\infty}^{x} \rho(\xi) \mathrm{d} \xi \quad \text { and } \quad \mathcal{R}_{n}(x)=\int_{-\infty}^{x} \rho_{n}(\xi) \mathrm{d} \xi
$$

satisfy $\mathcal{R}\left(p_{1}\right)=\mathcal{R}_{n}\left(p_{1}\right)=0$. Hence, since by (2.2) we have

$$
\int_{p_{i}}^{p_{i+1}} \rho(x) \mathrm{d} x=\int_{p_{i}}^{p_{i+1}} \rho_{n}(x) \mathrm{d} x=\ell_{n} \quad \text { for all } i=1, \ldots, n,
$$

we recursively obtain $\mathcal{R}\left(p_{i+1}\right)=\mathcal{R}_{n}\left(p_{i+1}\right)=i \ell_{n}$ for all $i=1, \ldots, n$.

By the monotonicity of $\mathcal{R}$ and $\mathcal{R}_{n}$, we have sup $\left|\mathcal{R}_{n}(x)-\mathcal{R}(x)\right| \leq \ell_{n}$ for every $i=1, \ldots, n$ and

$$
\sup _{x \in \mathbb{R}}\left|\mathcal{R}_{n}(x)-\mathcal{R}(x)\right| \leq \ell_{n}=\frac{m}{n},
$$


so that

$$
\begin{aligned}
\left|\int_{a}^{b} \rho_{n}(\xi) \mathrm{d} \xi-\int_{a}^{b} \rho(\xi) \mathrm{d} \xi\right| & =\left|\int_{a}^{b}\left(\rho_{n}(\xi)-\rho(\xi)\right) \mathrm{d} \xi\right| \\
& =\left|\left[\mathcal{R}_{n}(b)-\mathcal{R}(b)\right]-\left[\mathcal{R}_{n}(a)-\mathcal{R}(a)\right]\right| \\
& \leq\left|\mathcal{R}_{n}(b)-\mathcal{R}(b)\right|+\left|\mathcal{R}_{n}(a)-\mathcal{R}(a)\right| \\
& \leq 2 \sup _{x \in \mathbb{R}}\left|\mathcal{R}_{n}(x)-\mathcal{R}(x)\right| \\
& \leq 2 \frac{m}{n} \rightarrow 0 \quad \text { as } n \rightarrow+\infty
\end{aligned}
$$

completing step (I).

(II) $E$ is an open set with finite measure.

Write $E$ as disjoint union of intervals, i.e., $E=\dot{U}_{h \in \mathbb{N}} J_{h}$, see [9, Section 2.1, p. 27], and let $I_{k}=\bigcup_{h=0}^{k} J_{h}$. For any $\varepsilon>0$ there exists $k \in \mathbb{N}$ such that $\mathcal{L}^{1}\left(E \backslash I_{k}\right)<\frac{\varepsilon}{3}$. By step (I) above, there exists $\bar{n} \in \mathbb{N}$ such that for every $n \geq \bar{n}$

$$
\sum_{h=0}^{k}\left|\int_{J_{h}}\left(\rho_{n}(x)-\rho(x)\right) \mathrm{d} x\right|<\frac{\varepsilon}{3} .
$$

Compute preliminarily

$$
\begin{array}{r}
\left|\int_{I_{k}} \rho_{n}(x) \mathrm{d} x-\int_{I_{k}} \rho(x) \mathrm{d} x\right|=\left|\int \sum_{h=0}^{k} \chi_{J_{h}}\left(\rho_{n}(x)-\rho(x)\right) \mathrm{d} x\right| \leq \\
\sum_{h=0}^{k}\left|\int_{J_{h}}\left(\rho_{n}(x)-\rho(x)\right) \mathrm{d} x\right| .
\end{array}
$$

Hence, for every $n \geq \bar{n}$,

$$
\begin{aligned}
\left|\int_{E} \rho_{n}-\int_{E} \rho\right| & \leq\left|\int_{E} \rho_{n}-\int_{I_{k}} \rho_{n}\right|+\left|\int_{I_{k}} \rho_{n}-\int_{I_{k}} \rho\right|+\left|\int_{I_{k}} \rho-\int_{E} \rho\right| \\
& =\left|\int_{E} \rho_{n}\left(1-\chi_{I_{k}}\right)\right|+\left|\int_{I_{k}} \rho_{n}-\int_{I_{k}} \rho\right|+\left|\int_{E} \rho\left(1-\chi_{I_{k}}\right)\right| \\
& <\mathcal{L}^{1}\left(E \backslash I_{k}\right)+\frac{\varepsilon}{3}+\mathcal{L}^{1}\left(E \backslash I_{k}\right)<\varepsilon
\end{aligned}
$$

completing step (II). 
(III) $E$ has finite measure.

For every $\varepsilon>0$ there exists an open set $A$ such that $E \subseteq A$ and $\mathcal{L}^{1}(A \backslash E)<\varepsilon / 3$. By step (II) above there exists $\bar{n} \in \mathbb{N}$ such that for every $n \geq \bar{n}$

$$
\left|\int_{A} \rho_{n}(x) \mathrm{d} x-\int_{A} \rho(x) \mathrm{d} x\right|<\frac{\varepsilon}{3}
$$

Then, for every $n \geq \bar{n}$,

$$
\begin{aligned}
\left|\int_{E} \rho_{n}-\int_{E} \rho\right| & \leq\left|\int_{E} \rho_{n}-\int_{A} \rho_{n}\right|+\left|\int_{A} \rho_{n}-\int_{A} \rho\right|+\left|\int_{A} \rho-\int_{E} \rho\right| \\
& =\left|\int_{A} \rho_{n}\left(1-\chi_{E}\right)\right|+\left|\int_{A} \rho_{n}-\int_{A} \rho\right|+\left|\int_{A} \rho\left(1-\chi_{E}\right)\right| \\
& <\mathcal{L}^{1}(A \backslash E)+\frac{\varepsilon}{3}+\mathcal{L}^{1}(A \backslash E)<\varepsilon
\end{aligned}
$$

completing the proof.

Lemma 4.3. For every $m>0$, fix $\rho \in R_{m}$ and call $K=\operatorname{spt} \rho$. Then, $\left(C_{n} \circ E_{n}\right)(\rho) \rightarrow \rho$ in $\mathbf{L}^{1}(K, \mathbb{R})$ and, hence, in $\mathbf{L}^{1}(\mathbb{R}, \mathbb{R})$.

Proof. Denote $\rho_{n}=\left(C_{n} \circ E_{n}\right)(\rho)$. Let $\varphi \in \mathbf{L}^{\infty}(K, \mathbb{R})$ and $h \in \mathbb{N}, h>0$. Set

$$
\varphi_{h}=\sum_{i=-h+1}^{h+1} \frac{i}{h}\|\varphi\|_{\infty} \chi_{E_{i, h}} \quad \text { with } \quad E_{i, h}=\varphi^{-1}\left(\left[\frac{i-1}{h}\|\varphi\|_{\infty}, \frac{i}{h}\|\varphi\|_{\infty}[) .\right.\right.
$$

The $E_{i, h}$ have finite measure and, for fixed $h$, are pairwise disjoint. Moreover,

$$
\left\|\varphi-\varphi_{h}\right\|_{\mathbf{L}^{1}} \leq \mathcal{L}^{1}(K) \sup _{x \in K}\left|\varphi(x)-\varphi_{h}(x)\right| \leq \mathcal{L}^{1}(K) \frac{\|\varphi\|_{\infty}}{h}
$$

Fix $\varepsilon>0$ and choose $h$ so that $\left\|\varphi-\varphi_{h}\right\|_{\mathbf{L}^{1}}<\varepsilon$. Then, by Lemma 4.2, choose $\bar{n}$ so that

$$
\left|\int_{E_{i, h}} \rho_{n}(x) \mathrm{d} x-\int_{E_{i, h}} \rho(x) \mathrm{d} x\right|<\frac{\varepsilon}{h}
$$


for every $n \geq \bar{n}$. Hence

$$
\begin{aligned}
\left|\int \rho_{n} \varphi-\int \rho \varphi\right| & \leq\left|\int \rho_{n} \varphi-\int \rho_{n} \varphi_{h}\right|+\left|\int \rho_{n} \varphi_{h}-\int \rho \varphi_{h}\right|+\left|\int \rho \varphi_{h}-\int \rho \varphi\right| \\
& \leq\left\|\rho_{n}\right\|_{\infty}\left\|\varphi-\varphi_{h}\right\|_{\mathbf{L}^{1}}\left|+\int \varphi_{h}\left(\rho_{n}-\rho\right)\right|+\|\rho\|_{\infty}\left\|\varphi-\varphi_{h}\right\|_{\mathbf{L}^{1}} \\
& <\varepsilon+\left|\sum_{i=-h+1}^{h 1} \frac{i}{h}\|\varphi\|_{\infty} \int \rho_{E_{i, h}}\left(\rho_{n}-\rho\right)\right|+\varepsilon \\
& \leq 2 \varepsilon+\sum_{i=-h+1}^{h+1}\left|\frac{i}{h}\right|\|\varphi\|_{\infty}\left|\int_{E_{i, h}}\left(\rho_{n}-\rho\right)\right| \\
& <2 \varepsilon+\sum_{i=-h+1}^{h+1}\left|\frac{i}{h}\right| \frac{\varepsilon}{h}\|\varphi\|_{\infty} \\
& =\varepsilon\left(2+\left(1+\frac{1}{h}+\frac{1}{h^{2}}\right)\|\varphi\|_{\infty}\right) \\
& \leq \varepsilon\left(2+3\|\varphi\|_{\infty}\right),
\end{aligned}
$$

completing the proof.

Proof of Proposition 2.1. Consider first $E_{n} \circ C_{n}: P_{n} \rightarrow P_{n}$. Call $\boldsymbol{q}=\left(E_{n} \circ C_{n}\right)(\boldsymbol{p})$. By (2.3), spt $C_{n} \boldsymbol{p}=\left[p_{1}, p_{n+1}\left[\right.\right.$ and $\int_{\mathbb{R}} C_{n} \boldsymbol{p}(x) \mathrm{d} x=n \ell_{n}$. By (2.2), $q_{n+1}=\max \left(\operatorname{spt} C_{n} \boldsymbol{p}\right)=p_{n+1}$ and $q_{n}$ is such that

$$
\int_{q_{n}}^{p_{n+1}} C_{n} \boldsymbol{p}(x) \mathrm{d} x=\int_{q_{n}}^{q_{n+1}} C_{n} \boldsymbol{p}(x) \mathrm{d} x=\ell_{n}=\int_{p_{n}}^{p_{n+1}} \frac{\ell_{n}}{p_{n+1}-p_{n}} \mathrm{~d} x
$$

so that $q_{n}=p_{n}$ since $C_{n} \boldsymbol{p}$ is piecewise constant. Recursively $q_{i}=p_{i}$ for all $i=1, \ldots, n-1$, proving that $E_{n} \circ C_{n}=\operatorname{Id}_{P_{n}}$.

Consider now $C_{n} \circ E_{n}$. Let $\rho \in R_{m} \cap \mathbf{B V}(\mathbb{R} ;[0,1])$ and set $\left(C_{n} \circ E_{n}\right)(\rho)=\rho_{n}$.

By contradiction, suppose that $\rho_{n}$ does not converge strongly to $\rho$ in $\mathbf{L}^{1}$. This means that we can extract a subsequence $\left(\rho_{n_{k}}\right)$ such that $\left\|\rho_{n_{k}}-\rho\right\|_{\mathbf{L}^{1}}>\varepsilon$ for all $k$. Note that for all $k,\left\|\rho_{n_{k}}\right\|_{\mathbf{L}^{\infty}} \leq 1$ and, by Lemma 4.1, $\operatorname{TV}\left(\rho_{n_{k}}\right) \leq \operatorname{TV}(\rho)$. Then, Helly Theorem [2, Theorem 2.3] 
ensures that there exist a function $\rho_{*} \in\left(\mathbf{L}^{1} \cap \mathbf{B V}\right)(\mathbb{R} ;[0,1])$ and a $\left(\rho_{n_{k_{h}}}\right)$ such that $\lim _{h \rightarrow+\infty} \rho_{n_{k_{h}}}(x)=\rho_{*}(x)$ for every $x \in \mathbb{R}$. Remark that $\operatorname{spt} \rho_{n}=\operatorname{spt} \rho$ for all $n$ and hence, by the Dominated Convergence Theorem, $\rho_{n_{k_{h}}} \rightarrow \rho_{*}$ in $\mathbf{L}^{1}$. Lemma 4.3 then implies that $\rho_{*}=\rho$. This contradicts the choice of the subsequence $\rho_{n_{k}}$.

Proof of Proposition 2.3. The function $v$ is uniformly bounded and Lipschitz continuous. Extend it to a function with the same properties and defined on $\mathbb{R}^{+}$:

$$
u(\rho)= \begin{cases}v(\rho) & \text { if } \rho \in[0,1] \\ 0 & \text { if } \rho>1\end{cases}
$$

Note that the composition $\left\{\delta \mapsto u\left(\ell_{n} / \delta\right)\right\}$ can be extended to a uniformly bounded and Lipschitz continuous function on $\mathbb{R}^{+}$.

Consider the Cauchy problem

$$
\begin{cases}\dot{p}_{i}=u\left(\frac{\ell_{n}}{p_{i+1}-p_{i}}\right) & i=1, \ldots, n \\ \dot{p}_{n+1}=V & i=1, \ldots, n+1 . \\ p_{i}(0)=\tilde{p}_{i}\end{cases}
$$

By $(\mathbf{V})$ and the standard theory of Ordinary Differential Equations, there exists a $\mathbf{C}^{1}$ solution $\boldsymbol{p}$ defined as long as $p_{i+1}-p_{i}>0$ for all $i=1, \ldots, n$.

We prove now that for every $t \geq 0, p_{i+1}(t)-p_{i}(t) \geq \ell_{n}$, that is $\boldsymbol{p}(t) \in P_{n}$. Assume by contradiction that there exist positive $\bar{t}$ and $t^{*}$, with $\bar{t}<t^{*}$, such that

$$
\begin{aligned}
& p_{i+1}(\bar{t})-p_{i}(\bar{t})=\ell_{n}, \\
& \left.\left.p_{i+1}(t)-p_{i}(t)<\ell_{n} \quad \forall t \in\right] \bar{t}, t^{*}\right] .
\end{aligned}
$$

Then, for every $\left.t \in] \bar{t}, t^{*}\right]$ we have:

$$
p_{i}(t)=p_{i}(\bar{t})+\int_{\bar{t}}^{t} \dot{p}_{i}(s) \mathrm{d} s=p_{i}(\bar{t})+\int_{\bar{t}}^{t} u\left(\frac{\ell_{n}}{p_{i+1}(s)-p_{i}(s)}\right) \mathrm{d} s=p_{i}(\bar{t}) .
$$

Combining (4.2) and (4.1), we find $p_{i}(t)=p_{i}(\bar{t})$. This yields a contradiction, since for every $\left.t \in] \bar{t}, t^{*}\right], \ell_{n}>p_{i+1}(t)-p_{i}(t) \geq p_{i+1}(\bar{t})-p_{i}(\bar{t})=\ell_{n}$, completing the proof. 
Proof of Theorem 2.4. (We follow the general lines of the proof of [3, Proposition 4.2]). Proposition 2.3 implies that $\rho_{n}(t, x) \in[0,1]$ for every $t \in[0, T]$ and $x \in \mathbb{R}$. This holds also for its limit: $\rho(t, x) \in[0,1]$. Let $\Delta=\sup \operatorname{spt} \tilde{\rho}-\inf \operatorname{spt} \tilde{\rho}$. Recalling [2, Formula (4.5)], for any $\left.\left.\varphi \in \mathbf{C}_{c}^{1}(]-\infty, T\right] \times \mathbb{R} ; \mathbb{R}\right)$, compute

$$
\begin{aligned}
I_{n}:= & \int_{0}^{T} \int_{\mathbb{R}}\left(\rho_{n} \partial_{t} \varphi+\rho_{n} v\left(\rho_{n}\right) \partial_{x} \varphi\right) \mathrm{d} x \mathrm{~d} t+\int_{\mathbb{R}} \tilde{\rho}(x) \varphi(0, x) \mathrm{d} x \\
= & \sum_{i=1}^{n} \int_{0}^{T} \frac{\ell_{n}}{p_{i+1}(t)-p_{i}(t)} \int_{p_{i}(t)}^{p_{i+1}(t)}\left[\partial_{t} \varphi+v\left(\frac{\ell_{n}}{p_{i+1}(t)-p_{i}(t)}\right) \partial_{x} \varphi\right] \mathrm{d} x \mathrm{~d} t \\
& +\int_{\mathbb{R}} \rho_{n}(0, x) \varphi(0, x) \mathrm{d} x+\int_{\mathbb{R}}\left[\tilde{\rho}(x)-\rho_{n}(0, x)\right] \varphi(0, x) \mathrm{d} x \\
= & \sum_{i=1}^{n} \int_{0}^{T} \frac{\ell_{n}}{p_{i+1}(t)-p_{i}(t)} \int_{p_{i}(t)}^{p_{i+1}(t)}\left[\partial_{t} \varphi(t, x)+\dot{p}_{i}(t) \partial_{x} \varphi(t, x)\right] \mathrm{d} x \mathrm{~d} t \\
& +\sum_{i=1}^{n} \frac{\ell_{n}}{\tilde{p}_{i+1}-\tilde{p}_{i}} \int_{\tilde{p}_{i}} \varphi(0, x) \mathrm{d} x+\int_{\mathbb{R}}\left[\tilde{\rho}(x)-\rho_{n}(0, x)\right] \varphi(0, x) \mathrm{d} x .
\end{aligned}
$$

Now approximate $\varphi(t, x)$ with $\varphi\left(t, p_{i}(t)\right)$ for every $x$ in $\left[p_{i}(t), p_{i+1}(t)\right]$ and introduce the quantity $\|\varphi\|_{\mathbf{C}^{2}}$ that uniformly bounds from above the modulus of $\varphi$ and all its derivatives up to second order. Then, for $x \in\left[p_{i}(t), p_{i+1}(t)\right]$

$$
\left|\partial_{t} \varphi(t, x)+\dot{p}_{i}(t) \partial_{x} \varphi(t, x)-\frac{\mathrm{d}}{\mathrm{d} t} \varphi\left(t, p_{i}(t)\right)\right| \leq(1+V)\|\varphi\|_{\mathbf{C}^{2}}\left|p_{i+1}(t)-p_{i}(t)\right|,
$$

and, equivalently,

$$
\partial_{t} \varphi(t, x)+\dot{p}_{i}(t) \partial_{x} \varphi(t, x)=\frac{\mathrm{d}}{\mathrm{d} t} \varphi\left(t, p_{i}(t)\right)+\mathcal{O}(1)\left(p_{i+1}(t)-p_{i}(t)\right),
$$

where, in particular, $\mathcal{O}(1)$ depends neither on $p_{i}(t)$, nor on $i$, nor on $n$.

Using the latter estimate in the expression for $I_{n}$ above:

$$
\begin{aligned}
I_{n}= & \sum_{i=1}^{n} \int_{0}^{T} \frac{\ell_{n}}{p_{i+1}(t)-p_{i}(t)} \int_{p_{i}(t)}^{p_{i+1}(t)} \frac{\mathrm{d}}{\mathrm{d} t} \varphi\left(t, p_{i}(t)\right) \mathrm{d} x \mathrm{~d} t \\
& +\sum_{i=1}^{n} \int_{0}^{T} \frac{\ell_{n}}{p_{i+1}(t)-p_{i}(t)} \int_{p_{i}(t)}^{p_{i+1}(t)} \mathcal{O}(1)\left(p_{i+1}(t)-p_{i}(t)\right) \mathrm{d} x \mathrm{~d} t
\end{aligned}
$$




$$
\begin{aligned}
& +\sum_{i=1}^{n} \frac{\ell_{n}}{\tilde{p}_{i+1}-\tilde{p}_{i}} \int_{\tilde{p}_{i}}^{\tilde{p}_{i+1}} \varphi(0, x) \mathrm{d} x+\int_{\mathbb{R}}\left[\tilde{\rho}(x)-\rho_{n}(0, x)\right] \varphi(0, x) \mathrm{d} x \\
& =\ell_{n} \sum_{i=1}^{n} \int_{0}^{T} \frac{\mathrm{d}}{\mathrm{d} t} \varphi\left(t, p_{i}(t)\right) \mathrm{d} t+\ell_{n} \sum_{i=1}^{n} \int_{0}^{T} \mathcal{O}(1)\left(p_{i+1}(t)-p_{i}(t)\right) \mathrm{d} t \\
& +\sum_{i=1}^{n} \frac{\ell_{n}}{\tilde{p}_{i+1}-\tilde{p}_{i}} \int_{\tilde{p}_{i}}^{\tilde{p}_{i+1}} \varphi(0, x) \mathrm{d} x+\int_{\mathbb{R}}\left[\tilde{\rho}(x)-\rho_{n}(0, x)\right] \varphi(0, x) \mathrm{d} x \\
& =-\ell_{n} \sum_{i=1}^{n} \varphi\left(0, \tilde{p}_{i}\right)+\mathcal{O}(1) \ell_{n} \int_{0}^{T}\left(p_{n+1}(t)-p_{1}(t)\right) \mathrm{d} t \\
& +\sum_{i=1}^{n} \frac{\ell_{n}}{\tilde{p}_{i+1}-\tilde{p}_{i}} \int_{\tilde{p}_{i}}^{\tilde{p}_{i+1}} \varphi(0, x) \mathrm{d} x+\int_{\mathbb{R}}\left[\tilde{\rho}(x)-\rho_{n}(0, x)\right] \varphi(0, x) \mathrm{d} x \\
& =\sum_{i=1}^{n} \frac{\ell_{n}}{\tilde{p}_{i+1}-\tilde{p}_{i}} \int_{\tilde{p}_{i}}^{\tilde{p}_{i+1}}\left[\varphi(0, x)-\varphi\left(0, \tilde{p}_{i}\right)\right] \mathrm{d} x+\mathcal{O}(1) \ell_{n}(\Delta+V T) T \\
& +\int_{\mathbb{R}}\left[\tilde{\rho}(x)-\rho_{n}(0, x)\right] \varphi(0, x) \mathrm{d} x \\
& =\mathcal{O}(1) \ell_{n} \Delta+\mathcal{O}(1) \ell_{n}(\Delta+V T) T+\int_{\mathbb{R}}\left[\tilde{\rho}(x)-\rho_{n}(0, x)\right] \varphi(0, x) \mathrm{d} x .
\end{aligned}
$$

Since $\rho_{n}(0, x)=\tilde{\rho}_{n}(x)$ and $\tilde{\rho}_{n}=C_{n} \circ E_{n}(\tilde{\rho}) \rightarrow \tilde{\rho}$ (by Proposition 2.1) and $\ell_{n} \rightarrow 0$ (by definition), all the terms in the latter quantity vanish as $n \rightarrow+\infty$, completing the proof.

Acknowledgments. The authors thank Paola Goatin for useful discussions.

\section{REFERENCES}

[1] B. Argall, E. Cheleshin, J. M. Greenberg, C. Hinde and P.-J. Lin. A rigorous treatment of a follow-the-leader traffic model with traffic lights present. SIAM J. Appl. Math., 63(1):149-168 (electronic), 2002.

[2] A. Bressan. Hyperbolic systems of conservation laws, volume 20 of Oxford Lecture Series in Mathematics and its Applications. Oxford University Press, Oxford, 2000. The one-dimensional Cauchy problem. 
[3] R. M. Colombo, F. Marcellini and M. Rascle. A 2-phase traffic model based on a speed bound. SIAM J. Appl. Math., 70(7):2652-2666, 2010.

[4] R. M. Colombo and A. Marson. A Hólder continuous ODE related to traffic flow. Proc. Roy. Soc. Edinburgh Sect. A, 133(4):759-772, 2003.

[5] G. Costeseque. Analyse et modelisation du trafic routier: Passage du microscopique au macroscopique. Master's thesis, Ecole des Ponts ParisTech, 2011.

[6] R. J. LeVEQUE. Finite volume methods for hyperbolic problems. Cambridge Texts in Applied Mathematics. Cambridge University Press, Cambridge, 2002.

[7] M. J. Lighthill and G. B. Whitham. On kinematic waves. II. A theory of traffic flow on long crowded roads. Proc. Roy. Soc. London. Ser. A., 229:317345, 1955.

[8] K. Radhakrishnan and A. C. Hindmarsh. Description and Use of LSODE, the Livermore Solver for Ordinary Differential Equations. LLNL report UCRL-ID-113855. National Aeronautics and Space Administration, Lewis Research Center, 1993.

[9] M. M. RAo. Measure theory and integration. Pure and Applied Mathematics (New York). John Wiley \& Sons Inc., New York, 1987. A Wiley-Interscience Publication.

[10] P. I. RichaRds. Shock waves on the highway. Operations Res., 4:42-51, 1956.

[11] E. Rossi. On the micro-macro limit in traffic flow. Master's thesis, Università Cattolica del Sacro Cuore, Brescia, 2012.

[12] Transportation Research Board of the National Academies. 75 Years of the Fundamental Diagram for Traffic Flow Theory, Greenshields Symposium, Transportation Research Circular E-C149, Washington, June 2011.

Manoscritto pervenuto in redazione il 18 Febbraio 2013. 
